NEUROLOGIST STRATEGIES FOR OPTIMIZING THE PARKINSON'S DISEASE CLINICAL ENCOUNTER

R. Schwartz ${ }^{1}$, R. Trivedi ${ }^{2}$, K. Lorenz ${ }^{3}$, D. Zulman ${ }^{4}, 1$. VA Palo Alto Health Care System \& Stanford School of Medicine, 2. VA Palo Alto Health Care System, 3. Stanford School of Medicine, 4. Stanford University School of Medicine

Controlling symptoms and promoting quality of life for Parkinson's disease patients requires effective information exchange during neurology encounters. Objective: To characterize strategies neurologists use to educate patients, improve medication management, optimize communication, and address unmet needs. Methods: We conducted hour-long, in-person, semi-structured interviews with sixteen neurologists from four health care delivery systems. Interviews were designed to identify strategies neurologists use to improve patient education around medication and disease self-management, innovative clinical communication strategies, and opportunities to deliver care that better meets patient needs. Results: Four themes emerged 1) Neurologists perceived a need for structured tools to prompt identifying and tracking symptoms; 2) Because patients struggled to causally associate their symptoms with the disease or medication treatment, non-motor symptoms, especially hallucinations, required patient education to improve reporting; 3) Systems-level barriers to effective care delivery included the lack of an identified referral network of specialists, particularly for physical therapy and psychosocial distress, as well as time constraints that limited neurologists' ability to address all patient concerns; 4) Neurologists highlighted their specific skill deficits in leading conversations around end-of-life planning. Conclusions: Neurologists identified specific, actionable barriers to improving care delivery for Parkinson's disease patients. Integrating practice tools to prompt consideration of distressing symptoms, training for neurologists in communication around end-of-life care, and strengthening referral networks for rehabilitation and psychosocial support hold promise for improving care and quality of life for Parkinson's disease patients.

\section{PREDICTING CHANGES IN PHYSICAL ACTIVITY AND BLOOD PRESSURE AMONG OLDER ADULTS WITH HYPERTENSION}

M. Chen ${ }^{1}$, J. Hu ${ }^{2}$, T. McCoy ${ }^{3}$, S. Letvak ${ }^{4}$, L. Ivanov ${ }^{5}$, 1. Byrdine F. Lewis College of Nursing \& Health Professions, Georgia State University, 2. College of Nursing, Obio State University, 3. University of North Carolina at Greensboro, 4. School of Nursing, University of North Carolina at Greensboro, 5. College of Nursing, Chamberlain University

As many older Americans have uncontrolled high blood pressure, it is important to promote healthy lifestyles for older adults with hypertension. Regular physical activity is an effective way to prevent and control hypertension in older adults. However, older adults in the United States have low participation rates of regular physical activity. The purpose of this study was to test the predictors of changes in blood pressure and physical activity in community-dwelling older adults with hypertension. This study was a secondary analysis of a randomized controlled trial ( $\mathrm{N}=196)$. Participants were randomly assigned to the lifestyle intervention group $(n=103)$ or the wait-list control group $(n=93)$. Descriptive statistics and hierarchical multiple regression were performed to analyze characteristics of the sample and hypotheses testing. The results indicated that the final regression models were statistically significant. The hierarchical multiple regression analyses revealed that systolic blood pressure (SBP) at baseline and monthly income were significant predictors for predicting change in SBP $(\mathrm{p}<.05)$; age and diastolic blood pressure (DBP) at baseline were significant predictors for predicting change in DBP $(\mathrm{p}<.05)$. For predicting change in physical activity frequency, physical activity frequency at baseline was the only significant predictor $(\mathrm{p}<.05)$. This study suggests that the associations between significant predictors, blood pressure, and physical activity should be considered when developing lifestyle interventions for older adults with hypertension.

\section{RESTRICTIVE VENTILATORY PATTERNS IN RESIDENTS OF CONTINUING CARE RETIREMENT COMMUNITIES}

C. Kovach, M. Taani, C. Evans, University of

Wisconsin-Milwaukee

Restrictive breathing is a biologically plausible and modifiable risk factor for pneumonia and functional decline. This study was undertaken to understand: a) the number of older adults in Continuing Care Retirement Communities with spirometry-confirmed restrictive ventilatory patterns (RVP); b) extrapulmonary factors associated with RVP; and c) whether RVP is associated with ambulation level. Using a descriptive correlational design, we measured RVP with a portable spirometer, muscle strength with a Jamar ${ }^{\circledR}$ digital dynamometer, thoracic kyphosis with a flexicurve ruler, and cognitive status with the Mini Mental Status Exam. Observational tools included the PAIN-AD that captures pain with movement and the Respiratory Distress Observation Scale. Smoking history was captured by self-report, body mass index was calculated with information in the medical record, and ambulation status was retrieved from the Minimum Data Set. Of 90 consenting, 23 people were unable to complete the pulmonary function testing. Restrictive ventilatory patterns were evident in 34 of the 65 participants $(52 \%)$. Dementia, respiratory distress, kyphosis, and lower muscle strength were statistically significantly related to RVP. Seventy percent of cases were coded correctly and $69 \%$ of those with a moderate or severe RVP were predicted correctly. Body mass index and musculoskeletal pain were not related to RVP. There was a statistically significant differences in ambulation between those with and without RVP. Designing interventions to address modifiable factors associated with restrictive breathing patterns may prevent respiratory infections, help people to achieve better lung health, and increase physical activity tolerance.

\section{RICHMOND HEALTH AND WELLNESS PROGRAM: COMMUNITY ADVISORY COUNCIL OUTCOMES}

F. Zanjani ${ }^{1}$, K. Falls ${ }^{1}$, E. McQueen Gibson ${ }^{2}$, K. Patel ${ }^{2}$, E. Price ${ }^{2}$, L. Sargent ${ }^{3}$, P. Slattum ${ }^{4}$, P. Parsons ${ }^{1}$, 1. Virginia 